\title{
A comparative study on knowledge towards COVID-19 prevention among undergraduate students in Macao and Zhuhai, China
}

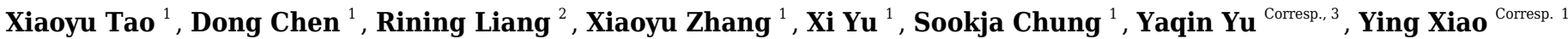 \\ 1 Faculty of Medicine, Macau University of Science and Technology, Macao, China \\ 2 Students' affairs division, Zhuhai College of Science and Technology, Zhuhai, Guangdong, China \\ 3 Faculty of Health Sciences, Zhuhai College of Science and Technology, Zhuhai, Guangdong, China \\ Corresponding Authors: Yaqin Yu, Ying Xiao \\ Email address: yuyaqin5540@163.com, yxiao@must.edu.mo
}

In order to strategy more suitable campus-based coronavirus disease 2019 (COVID-19) prevention programs for undergraduate students, it is critical to identify discrepancies in knowledge of COVID-19 prevention among students from different campuses in China. The present study examined the difference in preventive knowledge about COVID-19 pandemic in undergraduate students from two cities of Greater Bay Area (GBA), Macao Special Administrative Region (SAR), which had very few cases of COVID-19 patients, and Zhuhai, which is borders Macao SAR. In August 2020, two cohorts of undergraduate students from universities in Macao $(n=977)$ and Zhuhai $(n=2818)$ were recruited for online, self-rating questionnaire to gain information about their knowledge in COVID-19 prevention. Macao and Zhuhai students had similar correct rates in terms of heat inactivation conditions of COVID-19 virus, SARS-CoV-2, (76.8\% vs. $76.9 \%, P=0.950)$, etiquette when coughing and sneezing (75.9\% vs. $75.0 \%, P=0.562)$, and use of disposable masks $(92.2 \%$ vs. $90.6 \%$, $\mathrm{P}=0.126)$. However, students from Macao had significantly higher rates in correct use of disinfectants against SARS-CoV-2 $(24.6 \%$ vs. $17.5 \%, \mathrm{P}<0.001)$ and in the route of transmission of SARS-CoV-2 (84.5\% vs. $79.6 \%, \mathrm{P}<0.001)$ than those from Zhuhai. In conclusion, the knowledge level of COVID-19 prevention differs among undergraduate students from Macau and Zhuhai, which warrants an appropriate region-specific health education strategie for COVID-19 prevention. 
1 A comparative study on knowledge towards COVID-19 prevention among

2 undergraduate students in Macao and Zhuhai, China

3

4 Xiaoyu Tao ${ }^{1}$, Dong Chen ${ }^{1}$, Rining Liang ${ }^{2}$, Xiaoyu Zhang ${ }^{1}$, Xi Yu ${ }^{1}$, Sookja Chung ${ }^{1}$, Yaqin $\mathrm{Yu}^{3 *}$, 5 Ying $\mathrm{Xiao}^{1 *}$

6

$7 \quad$ I Faculty of Medicine, Macau University of Science and Technology, Taipa, Macao SAR, China

8 'Students' affairs division, Zhuhai College of Science and Technology, Zhuhai, China

$9{ }^{3}$ Faculty of Health Sciences, Zhuhai College of Science and Technology, Zhuhai, China

11 Abstract

12 In order to develop the strategy more suitable campus-based coronavirus disease 2019 (COVID-

13 19) prevention programs for undergraduate students, it is critical to identify discrepancies in 14 knowledge of COVID-19 prevention among students from different campuses in China. The 15 present study examined the difference in preventive knowledge about COVID-19 pandemic in 16 undergraduate students from two cities of Guangdong-Hong Kong-Macao Greater Bay Area 17 (GBA), Macao Special Administrative Region (SAR), which had very few cases of COVID-19 18 patients, and Zhuhai, which is borders Macao SAR. In August 2020, two cohorts of undergraduate

\footnotetext{
* Corresponding Author:

Prof. Ying Xiao, Faculty of Medicine, Macau University of Science and Technology, Macau SAR, China.

Tel: +85388972607

E-mail: yxiao@must.edu.mo

Prof. Yaqin Yu, Faculty of Health Sciences, Zhuhai College of Science and Technology, Zhuhai, Guangdong, China

Tel: +86(756)7626016

E-mail: yuyaqin5540@163.com
} 
19 students from universities in Macao $(n=977)$ and Zhuhai $(n=2818)$ were recruited for online. The

20 self-rating questionnaire was used to gain information about their knowledge in COVID-19

21 prevention. Macao and Zhuhai students had similar correct rates in terms of heat inactivation

22 conditions of SARS-CoV-2, (76.8\% vs. $76.9 \%, \mathrm{P}=0.950)$, etiquette when coughing and sneezing

$23(75.9 \%$ vs. $75.0 \%, \mathrm{P}=0.562)$, and use of disposable masks $(92.2 \%$ vs. $90.6 \%, \mathrm{P}=0.126)$. However,

24 students from Macao had significantly higher rates in correct use of disinfectants against SARS-

$25 \mathrm{CoV}-2(24.6 \%$ vs. $17.5 \%, \mathrm{P}<0.001)$ and in the route of transmission of SARS-CoV-2 (84.5\% vs.

$2679.6 \%, \mathrm{P}<0.001)$ than those from Zhuhai. In conclusion, the knowledge level of COVID-19

27 prevention differs among undergraduate students from Macau and Zhuhai, which warrants an

28 appropriate region-specific health education strategie for COVID-19 prevention.

29

30 Key words: knowledge, COVID-19, undergraduate student, Macao, Zhuhai 


\section{Introduction}

32 The Coronavirus Disease 2019 (COVID-19) pandemic is still posing a global health threat with

33 a sudden spurt of cases in some regions, such as India, U.S.A and UK. In China, there has been

34 some small-size outbreaks in its northern parts, such as Shijiazhuang municipality and Wangkui

35 county, recently, although the pandemic has been well controlled in Chia since May, 2020. At

36 present, COVID-19 vaccines are only allowed for emergency use in high-risk populations, such as

37 the staff of the departments of Disease Control and Prevention Centers and front-line healthcare

38 professionals in China. Therefore, precautionary measures are still the most effective way to

39 prevent the viral infection of COVID-19 among the general population (Chinese Center for

40 Disease Control and Prevention, 2020). As recommended by World Health Organization (WHO),

41 effective preventive measures include social distancing, quarantining, hand washing, and wearing

42 disposable medical masks (World Health Organization, 2020).

43 In China, universities have resumed zoom, face-to-face and/or hybrid classes after the successful 44 containment of COVID-19 in fall of 2020 (Ministry of Education of the People's Republic of

45 China, 2020). However, it should be noted that the risk of COVID-19 cluster outbreak is still

46 potentially high, since universities have large number of students in confound areas, such as small

47 classrooms, shared rooms in dormitories and common facilities (Zhai et al., 2020). Therefore, it is

48 of particularly importance to have students maintain correct precautionary behaviors during the

49 outbreak and post-outbreak periods. To this end, it is necessary to assess students' knowledge of

50 COVID-19 prevention, since there is evidence that good knowledge of COVID-19 preventive

51 measures is associated with appropriate practices towards COVID-19 outbreak (Zhong et al., 52 2020).

53 Macao Special Administrative Region (SAR) and Zhuhai are two major bordering

54 municipalities in Guangdong-Hong Kong-Macao Greater Bay Area (GBA) with distinct cultural, 
55 socio-economic, and educational backgrounds, as well as COVID-19 control measures.

56 Universities in Macao also have resumed classes since fall of 2020 (Education and Youth

57 Development Bureau, 2020), so universities of both municipalities face similar challenges of 58 COVID-19 prevention. The differences in the knowledge level of COVID-19 prevention in 59 undergraduate students between two municipalities and identification of underlying reasons for 60 such differences may facilitate the development of more suitable campus-based COVID-19 61 prevention programs. Therefore, the present study compared Macao and Zhuhai undergraduate 62 students' knowledge of COVID-19 prevention measures.

63

64 Methods

\section{Participants and procedures}

66 This cross-sectional survey was conducted in August, 2020 before the commencement of the 67 fall semester. Recruitment of participants and data collection were conducted online, because the 68 field survey was not feasible due to online courses for university students of the two municipalities.

69 The survey questionnaire was distributed via Wenjuanxing platform.

70 Several undergraduate students in Zhuhai and Macao were selected as primary seeds for this

71 survey and participated in making local network to invite other undergraduate peers. The sampling 72 approach is snowballing sampling. International students were not involved in the survey process.

73 Subjects, who agreed to participate in the survey were instructed to click the "Agree to 74 participate" button and then directed them to complete the anonymous self-administered 75 questionnaire.

76 Ethical clearance was obtained from the Ethical Review Committee of the Macau University of

77 Science and Technology affiliated Zhuhai Science Research Academy (Approval NO. MUST78 MEC-20200701XY). 


\section{Measures}

82 The COVID-19 preventive knowledge inventory (including the version of simplified Chinese 83 and traditional Chinese) was adapted from the questionnaire on Chinese residents' knowledge 84 towards COVID-19 (Table 1) (Panjin Municipal People's Government, 2020). It had five single85 choice questions on heat inactivation conditions for SARS-CoV-2, disinfectants for SARS-CoV86 2, transmission ways of SARS-CoV-2, etiquette when coughing and sneezing, and use of 87 disposable masks.

88 The survey questionnaire also collected demographic variables, including gender, age, and 89 type of students (medical vs. non-medical).

\section{Statistical analysis}

92 Correct rates of COVID-19 preventive knowledge were calculated for Macao and Zhuhai 93 students, and compared by using Chi-square test. Because the Macao and Zhuhai samples were 94 not comparable in terms of demographics, multiple logistic regression analyses were used to 95 examine the independent region-knowledge association, which included the answer to each

96 knowledge question (correct/incorrect) as the dependent variable, region (Macao vs. Zhuhai) as

97 the main predictor, and demographic variable (including the age, gender and major of students) as 98 covariates. The statistical significance level was set at $p<0.05$ (two-tailed). Data were analyzed 99 using the Statistical Package for the Social Sciences (SPSS 24.0).

\section{Results}

\section{Characteristics of the study population}


103 A total of 3,840 participants took part in the survey initially, but 3,795 (98.8\%) completed the

104 questionnaire, with 977 from Macao and 2818 from Zhuhai. As shown in Table 2, in comparison

105 to Zhuhai participants, Macao participants were overrepresented by male students with age

106 younger than 20 years and medical students.

107

108 Correct rates of COVID-19 preventive knowledge

109 As displayed in Table 3, Macao and Zhuhai participants had similar correct rates in terms of

110 heat inactivation conditions for SARS-CoV-2 (76.8\% vs. 76.9\%, $\mathrm{P}=0.950)$, etiquette when

111 coughing and sneezing $(75.9 \%$ vs. $75.0 \%, \mathrm{P}=0.562)$, and use of disposable masks $(92.2 \%$ vs.

$11290.6 \%, \mathrm{P}=0.126$ ), while Macao sample had significantly higher correct rates of disinfectants for

113 SARS-CoV-2 (24.6\% vs. $17.5 \%, \mathrm{P}<0.001)$ and transmission ways of SARS-CoV-2 $(84.5 \%$ vs.

$11479.6 \%, \mathrm{P}<0.001)$ than Zhuhai sample.

115 After adjusting for demographic variables (Table 4), the regional differences in correct rates of

116 disinfectants for SARS-CoV-2 $(\mathrm{P}<0.001)$ and transmission ways of SARS-CoV-2 $(\mathrm{P}=0.001)$

117 remained statistically significant.

118 


\section{Discussion}

120 This study compared university students' knowledge of COVID-19 prevention between Macao

121 and Zhuhai. Students of both cities had more than $75 \%$ rate of correct knowledge of heat

122 inactivation conditions for SARS-CoV-2, transmission ways of SARS-CoV-2, etiquette when

123 coughing and sneezing, and use of disposable masks. Although Macao students had statistically

124 significant higher correct rates of disinfectants for SARS-CoV-2 and transmission ways of SARS-

125 CoV-2 than Zhuhai students, which remained statistically significant after demographic variables

126 including age, gender, and types of students were adjusted, the correct rates of disinfectants were

127 still low in students of both cities. Our study results were similar to previous studies (Chang, Yuan,

128 \& Wang, 2020) (Puspitasari et al., 2020).

129 The higher percentage of medical students in Macao than Zhuhai students cannot fully explain

130 the regional difference in knowledge of disinfectants for SARS-CoV-2 and transmission ways of

131 SARS-CoV-2. The possible reason may be the students in Macao have more hygienic habits, such

132 as wearing masks, good etiquette when coughing and sneezing, and ventilating. Nevertheless, the

133 correct rates of disinfectants for SARS-CoV-2 were low in both cities, because the use of

134 disinfectants requires professional knowledge and lack of students' knowledge about disinfectants.

135 The present study has some limitations. First, the two university students were recruited online,

136 so the representation of participants might be limited and need to take caution in generalizing the

137 findings from the current study. Second, the knowledge measures covered in the questionnaire

138 are limited. Items, such as social distancing and hand hygiene, were not included. Finally, although

139 three possible confounding factors were adjusted, the possibility of residual confounding could not

140 be excluded.

141 Still, findings of the present studies suggest that health education programs are warranted to

142 further improve undergraduates' knowledge of COVID-19 prevention because approximately 10- 
$14315 \%$ (Macau SAR) and 25\% (Zhuhai) of the students of the two cities were unaware of the

144 transmission ways of SARS-CoV-2 and correct etiquette when coughing and sneezing,

145 respectively. There is also a need to educate students on the correct use of disinfection products

146 during the pandemic. Given that opinion leaders and role models appear to have a key function

147 among students (Abdi \& Simbar, 2013), the peer education approach based on social media

148 platforms may be an ideal form of health education among university students. Popular social

149 media platforms, such as WeChat, TikTok, and Weibo, may be a good avenue to spread correct

150 COVID-19 preventive knowledge, similar conclusion drawn in previous study in Pakistani

151 university populations (Salman et al., 2020).

152

\section{Acknowledgements}

154 This work was supported by the two universities in Zhuhai, Guangdong Province and Macao

155 SAR. We would like to thank all the investigators for collecting data and acknowledge all the

156 participants in our study. Also, we express our heartfelt respect to all the healthcare workers who

157 are fighting the epidemic on the front line.

158

159 Funding source

160 This study was supported by funding from Fundo do Ensino Superior of Macau (Grant No. HSS-

161 MUST-2020-6).

162

\section{Declaration of Competing Interest}

164 The authors have declared that no competing interest exists. 


\section{References}

167 1. Abdi F, Simbar M. 2013. The Peer Education Approach in Adolescents- Narrative Review 168 Article. Iranian Journal of Public Health 42:1200-1206.

169 2. Chinese Center for Disease Control and Prevention. 2020.Coronavirus Disease 2019.

$170 \quad$ Available at http://www.chinacdc.cn/jkzt/crb/zl/szkb_11803/(accessed February 4, 2021).

171 3. Chang, J., Yuan, Y., \& Wang, D. (2020). Nan fang yi ke da xue xue bao = Journal of

172 Southern Medical University, 40(2), 171-176. https://doi.org/10.12122/j.issn.1673-

$173 \quad 4254.2020 .02 .06$

174 4. Education and Youth Development Bureau (Government of Macao S.A.R.). 2020.Staff

175 from the DSEDJ visited the local school to understand the preparation work for the

$176 \quad$ 2020/2021 autumn semester. Available at

177 https://portal.dsedj.gov.mo/webdsejspace/internet/Inter_main_page.jsp?id=77814

$178 \quad$ (accessed February 4, 2021).

179 5. Ministry of Education of the People's Republic of China. 2020.Adhere to the principle of

180 "safe, normal, and comprehensive", prevent and control the pandemic scientifically and

181 accurately, and promote the start of the autumn semester steadily and orderly. Available at

182 http://www.moe.gov.cn/fbh/live/2020/52320/sfcl/202008/t20200827_480442.html (accessed

$183 \quad$ February 4, 2021).

184 6. Panjin Municipal People’s Government. 2020.2020 Unified Examination for Prevention and

185 Control of COVID-19 (China). Available at http://www.panjin.gov.cn/index.php/survey-

186 post-id-12 (accessed February 4, 2021).

187 7. Puspitasari, I. M., Yusuf, L., Sinuraya, R. K., Abdulah, R., \& Koyama, H. (2020).

188 Knowledge, Attitude, and Practice During the COVID-19 Pandemic: A Review. Journal of

189 multidisciplinary healthcare, 13, 727-733. https://doi.org/10.2147/JMDH.S265527 
190 8. Salman, M., Mustafa, Z. U., Asif, N., Zaidi, H. A., Hussain, K., Shehzadi, N., Khan, T. M.,

191 \& Saleem, Z. (2020). Knowledge, attitude and preventive practices related to COVID-19: a

192 cross-sectional study in two Pakistani university populations. Drugs \& therapy perspectives

193 : for rational drug selection and use, 1-7. Advance online publication.

194 https://doi.org/10.1007/s40267-020-00737-7

195 9. World Health Organization. 2020.Coronavirus disease (COVID-19). Available at

196 https://www.who.int/emergencies/diseases/novel-coronavirus-2019 (accessed February 4, 197 2021).

198 10. Zhai P, Ding Y, Wu X, Long J, Zhong Y, Li Y. 2020. The epidemiology, diagnosis and 199 treatment of COVID-19. International Journal of Antimicrobial Agents 55:105955. DOI:

$200 \quad$ 10.1016/j.ijantimicag.2020.105955.

201 11. Zhong B-L, Luo W, Li H-M, Zhang Q-Q, Liu X-G, Li W-T, Li Y. 2020. Knowledge, 202 attitudes, and practices towards COVID-19 among Chinese residents during the rapid rise 203 period of the COVID-19 outbreak: a quick online cross-sectional survey. International 204 Journal of Biological Sciences 16:1745-1752. DOI: 10.7150/ijbs.45221. 
Table $\mathbf{1}$ (on next page)

Table 1. Questionnaire of COVID-19 preventive knowledge 


\section{Table 1. Questionnaire of COVID-19 preventive knowledge}

Question list

Question 1: The time condition and temperature condition that can inactivate the SARS-CoV-2.

Option A. Maintain $56^{\circ} \mathrm{C}$ for 30 minutes

Option B. Maintain $56^{\circ} \mathrm{C}$ for 15 minutes

Option C. Maintain $56^{\circ} \mathrm{C}$ for 20 minutes

Option D. Maintain below $0{ }^{\circ} \mathrm{C}$ for 10 minutes

Question 2: The type of disinfectant that cannot inactivate the SARS-CoV-2.

Option A. Peroxyacetic acid

Option B. 75\% ethanol

Option C. Iodophor

Option D. Bleach powder

Question 3: The non-transmission pathways of the SARS-CoV-2.

Option A. Contact transmission

Option B. Droplet transmission

Option C. Soil transmission

Option D. Aerosol transmission

Question 4: The wrong precaution when coughing and sneezing.

Option A. Cover your nose and mouth with a tissue or elbow when coughing and sneezing.

Option B. Cover your nose and mouth with both hands when coughing and sneezing.

Option C. Put the sneezing tissue in the dustbin with cover.

Option D. It's best to clean your hands thoroughly after sneezing and coughing.

Question 5: The wrong precaution for the use of disposable medical masks.

Option A. It is recommended to replace the mask every 2-4 hours.

Option B. It should be replaced immediately once the mask has contaminated.

Option C. When wearing masks, avoid touching the inner side of the mask.

Option D. The thicker the mask, the better the anti-virus effect. 
Table 2 (on next page)

Table 2. Characteristics of the respondent 
1 Table 2. Characteristics of the respondent

\begin{tabular}{|c|c|c|c|c|}
\hline \multirow[b]{2}{*}{ Variable } & \multicolumn{2}{|c|}{$\mathrm{n}(\%)$} & \multirow[b]{2}{*}{$\chi^{2}$-value } & \multirow[b]{2}{*}{$P$-value } \\
\hline & $\begin{array}{l}\text { Macao } \\
(\mathrm{n}=977)\end{array}$ & $\begin{array}{c}\text { Zhuhai } \\
(\mathrm{n}=2818)\end{array}$ & & \\
\hline \multicolumn{5}{|l|}{ Gender } \\
\hline Female & $530(54.2 \%)$ & $1663(59.0 \%)$ & \multirow{2}{*}{6.755} & \multirow{2}{*}{0.009} \\
\hline Male & $447(45.8 \%)$ & $1155(41.0 \%)$ & & \\
\hline \multicolumn{5}{|l|}{ Age } \\
\hline$<20$ & $355(36.3 \%)$ & $602(21.4 \%)$ & \multirow{2}{*}{86.247} & \multirow{2}{*}{$<0.001$} \\
\hline$\geq 20$ & $622(63.7 \%)$ & $2216(78.6 \%)$ & & \\
\hline \multicolumn{5}{|l|}{ Major } \\
\hline Medical & $217(22.2 \%)$ & $268(9.5 \%)$ & \multirow{2}{*}{104.984} & \multirow{2}{*}{$<0.001$} \\
\hline Non-medical & $760(77.8 \%)$ & $2550(90.5 \%)$ & & \\
\hline
\end{tabular}

2

3 


\section{Table 3(on next page)}

Table 3. Correct rates of COVID-19 preventive knowledge in undergraduates of Macao and Zhuhai, China 
1 Table 3. Correct rates of COVID-19 preventive knowledge in undergraduates of Macao and

2 Zhuhai, China

\begin{tabular}{|c|c|c|c|c|}
\hline \multirow{2}{*}{ Question subject } & \multicolumn{2}{|c|}{$\mathrm{n}(\%)$} & \multirow{2}{*}{$\chi^{2}$-value } & \multirow{2}{*}{$\begin{array}{c}P \text { - } \\
\text { value }\end{array}$} \\
\hline & Macao & Zhuhai & & \\
\hline Inactivation conditions for SARS-CoV-2 & $\begin{array}{c}750 \\
(76.8 \%)\end{array}$ & $2166(76.9 \%)$ & 0.004 & 0.950 \\
\hline Disinfectants for SARS-CoV-2 & $\begin{array}{c}240 \\
(24.6 \%)\end{array}$ & $493(17.5 \%)$ & 23.271 & $<0.001$ \\
\hline Transmission ways of SARS-CoV-2 & $\begin{array}{c}826 \\
(84.5 \%)\end{array}$ & $2244(79.6 \%)$ & 11.334 & 0.001 \\
\hline Etiquette when coughing and sneezing & $\begin{array}{c}742 \\
(75.9 \%)\end{array}$ & $2114(75.0 \%)$ & 0.336 & 0.562 \\
\hline Use of disposable masks & $\begin{array}{c}901 \\
(92.2 \%)\end{array}$ & $2553(90.6 \%)$ & 2.342 & 0.126 \\
\hline
\end{tabular}


Table 4 (on next page)

Table 4. Logistic regression on the association of correct COVID-19 knowledge with region, adjusted for demographic variables 
1 Table 4. Logistic regression on the association of correct COVID-19 knowledge with region, adjusted for demographic variables.

\begin{tabular}{|c|c|c|c|c|c|c|c|c|c|c|c|c|c|c|c|c|c|c|c|c|}
\hline \multirow{4}{*}{ Variable $^{\S}$} & \multicolumn{20}{|c|}{ Question subject } \\
\hline & \multicolumn{4}{|c|}{$\begin{array}{l}\text { Inactivation conditions for } \\
\text { SARS-CoV-2 }\end{array}$} & \multicolumn{4}{|c|}{$\begin{array}{l}\text { Disinfectants for } \\
\text { SARS-CoV-2 }\end{array}$} & \multicolumn{4}{|c|}{$\begin{array}{c}\text { Transmission ways of } \\
\text { SARS-CoV-2 }\end{array}$} & \multicolumn{4}{|c|}{$\begin{array}{c}\text { Etiquette when coughing } \\
\text { and sneezing }\end{array}$} & \multicolumn{4}{|c|}{ Use of disposable masks } \\
\hline & \multirow{2}{*}{$\mathrm{OR}^{*}$} & \multicolumn{2}{|c|}{$95 \% \mathrm{CI}^{\Delta}$} & \multirow{2}{*}{$\mathrm{P}$} & \multirow{2}{*}{ OR } & \multicolumn{2}{|c|}{$95 \% \mathrm{CI}$} & \multirow{2}{*}{$\mathrm{P}$} & \multirow{2}{*}{ OR } & \multicolumn{2}{|c|}{$95 \% \mathrm{CI}$} & \multirow{2}{*}{$\mathrm{P}$} & \multirow{2}{*}{ OR } & \multicolumn{2}{|c|}{$95 \% \mathrm{CI}$} & \multirow{2}{*}{$\mathrm{P}$} & \multirow{2}{*}{ OR } & \multicolumn{2}{|c|}{$95 \% \mathrm{CI}$} & \multirow{2}{*}{$\mathrm{P}$} \\
\hline & & upper & lower & & & upper & lower & & & upper & Lower & & & upper & lower & & & upper & lower & \\
\hline Region & 1.086 & 0.910 & 1.297 & 0.360 & 0.713 & 0.595 & 0.855 & $<0.001$ & 0.704 & 0.576 & 0.861 & 0.001 & 0.978 & 0.821 & 1.166 & 0.807 & 0.809 & 0.615 & 1.064 & 0.130 \\
\hline Age & 1.123 & 0.941 & 1.342 & 0.199 & 1.169 & 0.975 & 1.403 & 0.092 & 0.855 & 0.712 & 1.027 & 0.094 & 1.117 & 0.938 & 1.329 & 0.214 & 1.236 & 0.944 & 1.620 & 0.124 \\
\hline Gender & 1.022 & 0.877 & 1.191 & 0.777 & 1.123 & 0.951 & 1.325 & 0.172 & 0.754 & 0.638 & 0.891 & 0.001 & 1.671 & 1.440 & 1.939 & $<0.001$ & 2.123 & 1.692 & 2.663 & $<0.001$ \\
\hline $\begin{array}{l}\text { Type } \\
\text { of } \\
\text { stude } \\
\text { nts }\end{array}$ & 1.650 & 1.278 & 2.131 & $<0.001$ & 1.957 & 1.579 & 2.425 & $<0.001$ & 1.117 & 0.872 & 1.431 & 0.381 & 1.391 & 1.092 & 1.772 & 0.008 & 1.122 & 0.784 & 1.606 & 0.529 \\
\hline
\end{tabular}

2 § Region: $1=$ Zhuhai, $2=$ Macao; Age: $1=$ Less than 20 years old, $2=20$ years or older; Gender: $1=$ Female, $2=$ Male; Type of students:

3 1=Medical students, $2=$ Non-medical students

4 * OR: Odds Ratio

$5 \quad \Delta$ CI: Confidence Interval 